\title{
A Neural Mechanism Mediating the Impact of Episodic Prospection on Farsighted Decisions
}

\author{
Roland G. Benoit, ${ }^{1,2}$ Sam J. Gilbert, ${ }^{2}$ and Paul W. Burgess ${ }^{2}$ \\ ${ }^{1}$ Medical Research Council Cognition and Brain Sciences Unit, Cambridge CB2 7EF, United Kingdom, and ${ }^{2}$ Institute of Cognitive Neuroscience, University \\ College London, London WC1N 3AR, United Kingdom
}

Humans can vividly imagine possible future events. This faculty, episodic prospection, allows the simulation of distant outcomes and desires. Here, we provide evidence for the adaptive function of this capacity and elucidate its neuronal basis. Participants either imagined specific events of spending money (e.g., $\mathfrak{E} 35$ in 180 days at a pub), or merely estimated what the money could purchase in the scenario. Imagining the future biased subsequent monetary decisions toward choices associated with a higher long-term pay-off. It thus effectively attenuated temporal discounting, i.e., the propensity to devalue rewards with a delay until delivery. Using functional magnetic resonance imaging, we implicate the medial rostral prefrontal cortex (mrPFC) in this effect. Blood oxygen level-dependent signal in this region predicted future-oriented choices on a trial-by-trial basis. Activation reflected the reward magnitude of imagined episodes, and greater reward sensitivity was related to less discounting. This effect was also associated with increased mrPFC-hippocampal coupling. The data suggest that mrPFC uses information conveyed by the hippocampus to represent the undiscounted utility of envisaged events. The immediate experience of the delayed reward value might then bias toward farsighted decisions.

\section{Introduction}

Humans forego choices that would be more beneficial in the long run in favor of smaller but more immediate rewards. This tendency reflects a fundamental psychological principle: temporal discounting, i.e., the propensity to devalue a reward with its delay (Green and Myerson, 2004). However, humans can also vividly envisage possible future episodes (Suddendorf and Corballis, 2007). This faculty, episodic prospection, allows for the immediate experience of the event's anticipated affective impact. It therefore conveys its undiscounted reward value. In this way, imagining a future reward might add weight to its valuation, thus effectively attenuating temporal discounting (Berns et al., 2007; Boyer, 2008; Peters and Büchel, 2010).

How might this process be mediated by the brain? Neuroimaging studies have revealed a core network that is consistently engaged during episodic prospection (Hassabis and Maguire, 2007; Schacter et al., 2007; Spreng et al., 2009). While nodes such as the hippocampus (HC) seem to support the construction of spatially coherent events (Hassabis et al., 2007; Bird et al., 2010), the medial rostral prefrontal cortex (mrPFC) (approximating medial Brodmann area 10, extending into 9) appears to be primarily recruited

Received Dec. 14, 2010; revised Feb. 17, 2011; accepted March 8, 2011.

Author contributions: R.G.B., S.J.G., and P.W.B. designed research; R.G.B. performed research; R.G.B. and S.J.G. analyzed data; R.G.B., S.J.G., and P.W.B. wrote the paper.

R.G.B. is funded by the Medical Research Council, UK, and S.J.G. by the Royal Society. We thankF. Dick, O. Josephs, and M. Sereno for their technical help, G. Gonen-Yaacovi and E. Volle for their assistance in data collection, and B. Staresina for comments on an earlier draft of this manuscript.

Correspondence should be addressed to Roland G. Benoit, MRC Cognition and Brain Sciences Unit, 15 Chaucer Road, Cambridge CB2 7EF, UK. E-mail: roland.benoit@mrc-cbu.cam.ac.uk.

DOI:10.1523/JNEUROSCI.6559-10.2011

Copyright $\odot 2011$ the authors $\quad 0270-6474 / 11 / 316771-09 \$ 15.00 / 0$ when participants elaborate on already constructed scenes (Addis et al., 2007). Importantly, activation in this region and adjacent rostral anterior cingulate is stronger during envisioning positive rather than negative episodes (Sharot et al., 2007; D’Argembeau et al., 2008). These observations suggest that mrPFC recruitment reflects attributes of the imagined event. Here, we hypothesize that it represents reward values of envisaged episodes, thereby contributing to the putative impact of episodic prospection on discounting.

This account is consistent with emerging evidence implicating mrPFC in valuation processes (Montague et al., 2006; Rushworth and Behrens, 2008). Recent studies observed a tight coupling between blood oxygen level-dependent (BOLD) signal and the subjective value of monetary options that varied in magnitude and delay (Kable and Glimcher, 2007; Peters and Büchel, 2009). Similarly, activation in a more ventral region has been shown to vary with the subjective value of food items, as assessed by the willingness to pay for these goods (Plassmann et al., 2007). Accordingly, greater activation during the imagination of positive episodes (Sharot et al., 2007; D'Argembeau et al., 2008) might reflect their inherently greater subjective value. However, there is no direct evidence for a role of mrPFC in the undiscounted representation of envisaged rewards, and no functional link between such a representation and attenuated discounting has been established.

In this functional magnetic resonance imaging (fMRI) study, participants either imagined specific episodes of spending money (e.g., $\mathfrak{E} 35$ in 180 days at a pub), or merely estimated what the money could purchase in the scenarios. They then indicated their preference for the considered, delayed reward option (e.g., $\mathfrak{E} 35$ in 180 days) versus a smaller but immediate reward. We thus investigated (1) the influence of episodic prospection on temporal 
discounting, and (2) the neural basis of this effect: mrPFC might mediate the impact of episodic prospection by representing the reward magnitude of envisaged events. In this case, we expected a tight coupling between activation for imagined rewards and the episodic prospection effect.

\section{Materials and Methods}

Participants. Fifteen right-handed volunteers participated in this experiment. They all reported no history of psychiatric or neurological disorder, and gave written consent as approved by the local research ethics committee. Three participants had to be excluded due to miscomprehension of task instructions as revealed by debriefing (see below). Thus, data from 12 participants ( 4 males; mean age $=27.3$ years, range $=20.6-36.3$ years) are reported.

Tasks and procedure. Participants considered monetary rewards in different scenarios. The magnitude of the reward and the delay until its delivery were varied. They either imagined specific episodes of spending

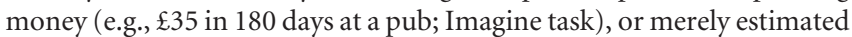
what it could purchase in the scenario (Estimate task). Thus, the tasks were similar in their semantic retrieval demands, at least insofar as both required knowledge of the goods associated with the specific scenarios as well as their typical prices. By contrast, only the Imagine task required episodic prospection, i.e., the mental experience of a potential future episode (Atance and O'Neill, 2001; Suddendorf and Corballis, 2007). Although piloting indicated that participants performed the Estimate task, as instructed, throughout the task period, we cannot rule out that they did not engage in episodic prospection at all. However, this could only have weakened any observed effects.

Before the fMRI session, participants were thoroughly familiarized with the tasks and 24 different scenarios (e.g., pub, dining out, museum, concert). They were presented with a list of possible items that one would purchase in the respective situations. Participants briefly imagined each item and guessed what it would typically cost. Afterward, they rated on a 5 -point scale how much they would enjoy being in the scenario. For the scanning session, those 20 situations were chosen that participants liked most. Familiarizing participants with the scenarios also minimized the semantic retrieval requirements of the two tasks. Therefore, they had relatively more time for elaborating rather than constructing the episodes (Addis et al., 2007).

Each trial started with the presentation of a task cue (i.e., imagine, estimate) and the scenario (e.g., pub) for $2 \mathrm{~s}$ (Fig. 1). Next, the delayed

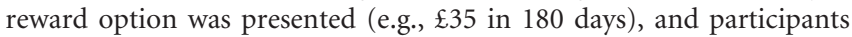
performed the task for $14 \mathrm{~s}$. An auditory signal then prompted participants to indicate their preference for either the reward option that they had just considered and which would only be delivered after a delay (e.g., $\mathfrak{E} 35$ in 180 days), or for a smaller but immediate reward ( $£ 25)$. Analyses included those 12 subjects who remembered that these choices were formally independent of the preceding task (e.g., $\mathfrak{£ 3 5}$ would not have to be spent in a pub). Previous research has shown that hypothetical and actual rewards lead to similar patterns of discounting (Johnson and Bickel, 2002; Madden et al., 2003), and also the functional neuroanatomy associated with the valuation of either kind of reward appears to be very similar (Bickel et al., 2009).

Following their decision, or after $4.5 \mathrm{~s}$, they rated on a 4-point scale (1) the emotional intensity that they had felt while performing the task (Addis et al., 2007), and (2) their feeling of mentally experiencing a specific episode (D'Argembeau et al., 2008). The ratings were self-paced (maximal $4 \mathrm{~s}$ each). A trial concluded with the presentation of a fixation cross that remained onscreen until $28.5 \mathrm{~s}$ following trial onset. Each task was performed with 20 different scenarios and all combinations of five reward magnitudes ( $£ 28,35,42,52$, and 65$)$ and four delays $(30,90,180$, and $360 \mathrm{~d}$ ). Piloting confirmed that the chosen levels of reward magni- tude and delay allowed for the imagination of phenomenologically different experiences. The assignment of the delayed options to the scenarios was random.

In addition, participants performed a counting task that did not require considering money. Specifically, they had to count either backwards or forwards from a given number in steps of 3 or 4 . This task allowed for the assessment of BOLD signal changes common to the Imagine and Estimate task while controlling for activation associated with basic arithmetic operations that are likely to occur in those tasks.

The three tasks alternated randomly, with the constraint that no task was immediately repeated. The session was divided into two functional runs, each comprising 10 trials of the Imagine and the Estimate task as well as 6 counting trials. Every scenario and each combination of amount and delay was used only once in each run. Participants were further instructed to keep their eyes opened and fixated at all times.

Debriefing corroborated that participants primarily engaged in episodic prospection in the Imagine task. They also reported that they had consistently imagined new, fictitious experiences rather than recollected autobiographical memories. Moreover, we checked that all participants had thought of the monetary choices as being formally independent of the preceding task. Finally, they completed the Consideration of Future Consequences questionnaire (Strathman et al., 1994), which assesses the proneness to focus on the distant versus immediate outcomes of one's actions in everyday life.

MRI data acquisition and preprocessing. A Siemens Avanto 1.5T MRI scanner was used for interleaved acquisition of T2*-weighted echoplanar images sensitive to BOLD contrast $[64 \times 64 ; 3.5 \times 3.5 \mathrm{~mm}$ pixels; repetition time (TR), $2.5 \mathrm{~s}$; echo time (TE), $45 \mathrm{~ms}$; flip angle $90^{\circ}$. The brain was covered by volumes that comprised 31 axial slices $(3.5 \mathrm{~mm}$ thick, oriented to the anterior-posterior commissure plane), omitting aspects of the superior parietal lobes. For each of the two sessions a separate functional scan of 302 volumes was acquired, of which the first 4 volumes were discarded to allow for T1 equilibration effects. Additionally, MPRAGE (magnetization-prepared rapid-acquisition gradient echo) structural images were acquired $\left(256 \times 256 ; 1 \mathrm{~mm}^{3}\right.$ cubic voxels; 176 slices; TR, $2730 \mathrm{~ms}$; TE, $3.57 \mathrm{~ms}$; flip angle $7^{\circ}$ ).

fMRI data were analyzed using SPM5 (http://www.fil.ion.ucl.ac.uk/ $\mathrm{spm} /$ software/spm5/). The volumes were corrected for different slice acquisition times, realigned, and coregistered with the structural images. These were spatially normalized, and the resulting parameters served to normalize the functional images into $3 \times 3 \times 3 \mathrm{~mm}^{3}$ cubic voxels by fourth-degree B-spine interpolation (using the Montreal Neurological Institute reference brain). The images were then smoothed by an isotropic $8 \mathrm{~mm}$ full-width half-maximal Gaussian kernel.

Statistical analyses. The variance in BOLD signal was decomposed in a general linear model (Friston et al., 1995), separately for each run. Sustained activation associated with the main task interval (i.e., onset 
with presentation of the delayed option; lasting for $14 \mathrm{~s}$ ) was modeled by boxcar regressors, which were convolved with the canonical hemodynamic response function (HRF). Separate regressors coded for the 10 combinations of task (Imagine, Estimate) and amount of money

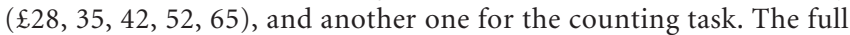
model additionally comprised regressors representing the mean over scans and residual movement artifacts. A $1 / 128 \mathrm{~Hz}$ high-pass filter was applied to the data and the model. Parameters for each regressor were estimated from the least-mean-squares fit of the model to the data. Effects of interest were assessed in a random-effects analysis. Contrast estimates for each of the 10 combinations of task and amount of money were entered into a repeated-measures ANOVA using non-sphericity correction. The task effect (imagine $>$ estimate) was defined as a contrast vector, where all 5 estimates of a given task were weighted equally. To assess the parametric modulation of the task effect by reward magnitudes, estimates were weighted by the mean-centered natural logarithm (ln) of the amount of money. The ln accounts for the diminishing marginal utility of increasing sums of money (Bernoulli, 1738; Pine et al., 2009).

To test whether the contrasts of (1) the tasks and (2) their respective parametric modulations by reward magnitude were associated with BOLD signal changes in overlapping regions, a conjunction analysis was performed using the minimum statistic approach (Nichols et al., 2005).

Moreover, a psychophysiological interaction (PPI) analysis was conducted (Friston et al., 1997) to examine mrPFC-hippocampal coupling during the Imagine task as a function of attenuated discounting. Separately for each subject, the mrPFC peak of the task contrast (imagine $>$ estimate) was identified that was closest to the group-level peak of the conjunction analysis $(-3,57,30$; all within 24 $\mathrm{mm}$ distance). These peaks served as centers for spherical regions of interest (ROIs) (radius $=5 \mathrm{~mm}$ ). The first eigenvariate from these ROIs (i.e., the first principal component of the time-series of the voxels) constituted the physiological variable. The psychological variable was defined as the contrast vector representing the task effect (imagine $>$ estimate). These regressors and their interaction term (i.e., the PPI regressor) were estimated at the first level. Contrast images associated with the PPI regressor were then entered into a regression analysis at the second level with the effect size of attenuated discounting as the explanatory variable (see Results).

An additional model was estimated to detect brain regions that exhibit greater BOLD signal during those trials of the Imagine task that are followed by choices of the delayed option. This subsequent choice analysis comprised four boxcar regressors, each of which was convolved with the canonical HRF. One regressor coded for the Estimate and another one for the Counting task. Two regressors coded for the Imagine task: one for those trials that were followed by choices of the delayed option, and one for the other trials which were followed by choices of the immediate option. To statistically control for the effect of reward magnitude within each condition (i.e., in the two Imagine conditions and in the Estimate task), three additional nuisance regressors coded for the linear parametric effect of reward magnitude in each condition (i.e., the mean-centered $\ln$ of the reward magnitudes). The full model additionally comprised regressors representing the mean over scans and residual movement artifacts. Finally, an analogous subsequent choice model was estimated for the Estimate task. One participant had to be excluded from this analysis due to constant choices of the delayed reward option following this task.

For whole-brain analyses, statistical parametric maps (SPMs) were thresholded at $p<0.001$, uncorrected, with a minimum cluster size of 10 contiguous voxels (i.e., a volume of $270 \mathrm{~mm}^{3}$ ). To assess the significance of the effects at a corrected threshold, ROIs were generated for mrPFC and the HC, using the WFU PickAtlas (Maldjian et al., 2003). The mrPFC mask comprised the medial parts of Brodmann areas 9 and 10 (defined as any region up to two-fifths of the distance from the midline to the lateral edge of the brain), dilated by three voxels. For the HC, the bilateral mask was used. Where noted in the text, activations survived small-volume corrections at the cluster level within these ROIs.

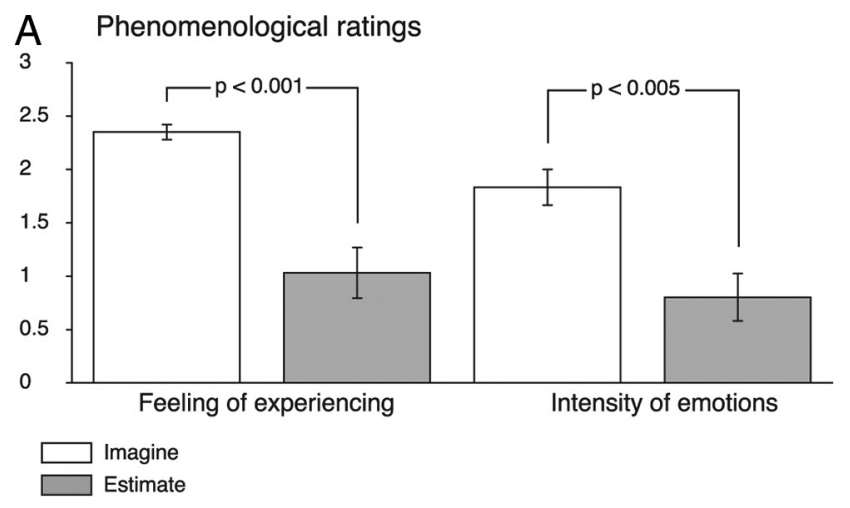

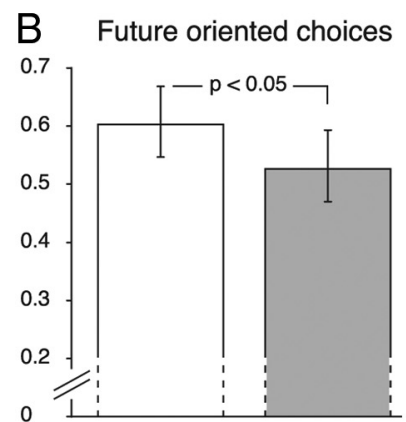

Choice index

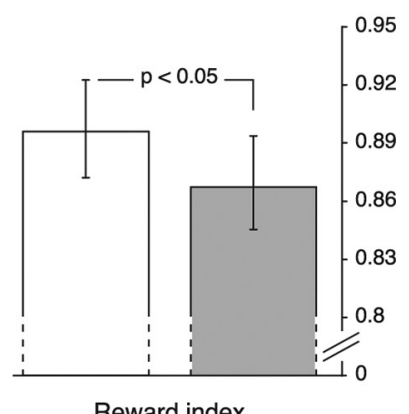

Reward index

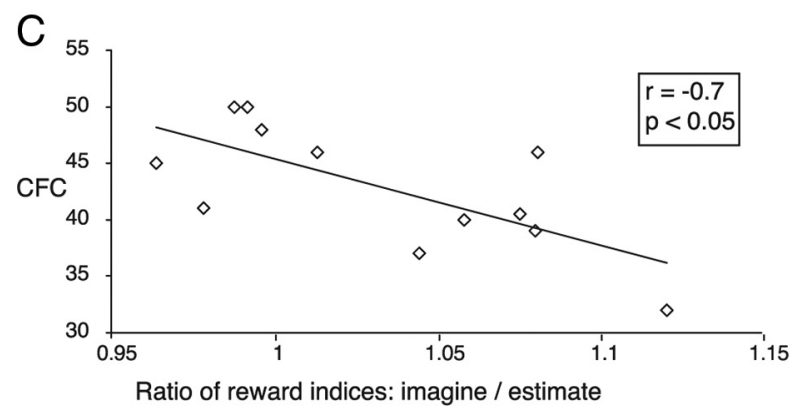

Figure 2. Behavioral results. $\boldsymbol{A}$, Phenomenological ratings for (1) the feeling of experiencing an episode and (2) the emotional intensity while imagining (white) or estimating (gray) ( $p$ values from two-tailed paired-samples $t$ tests). $\boldsymbol{B}$, Discounting behavior as quantified by the ratios of (1) the frequency of delayed options chosen versus all options chosen (i.e., delayed plus immediate) (choice index), and (2) the accumulated reward over trials versus the maximal possible reward (reward index) ( $p$ values from one-tailed $t$ tests). $C$, Correlation between the Consideration of Future Consequences scale (CFC) and the ratios of the reward indices for the Imagine versus the Estimate task ( $p$ value from two-tailed test). Error bars indicate the SEM.

\section{Results}

\section{Phenomenological ratings}

The phenomenological ratings confirmed that participants primarily engaged in episodic prospection during the Imagine task, since the intensity of experiencing an event was significantly greater than in the Estimate task $\left(t_{(11)}=5.45, p=0.0002\right.$; Fig. $2 A)$. Moreover, imagining spending the money elicited a stronger emotional intensity than estimating what it could purchase $\left(t_{(11)}=4.29, p=0.001\right.$; Fig. $\left.2 A\right)$.

\section{Episodic prospection attenuates temporal discounting}

Discounting was quantified by the ratios of (1) the frequency of delayed options chosen versus all options chosen (i.e., delayed plus immediate) ("choice index"), and (2) the accumulated reward over trials versus the maximal possible reward ("reward index") (Boettiger et al., 2007). The results were consistent with 
Table 1. Regions in which BOLD signal was significantly greater in the Imagine versus the Estimate task

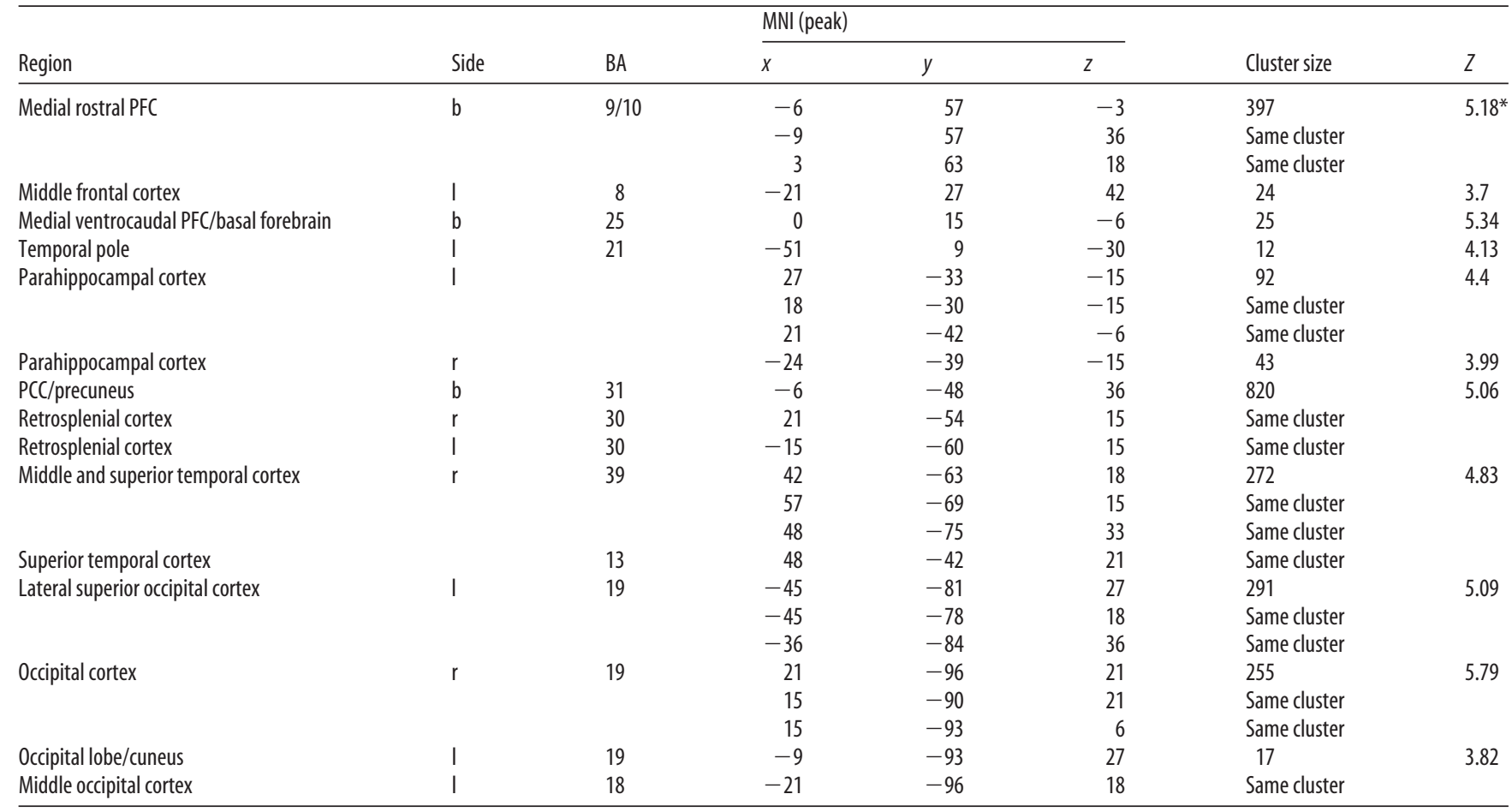

Thresholded at $p<0.001$, uncorrected, with a minimum cluster size of 10 contiguous voxels. Brodmann areas (BA) are approximate. b, Bilateral; l, left; r, right; PCC, posterior cingulate cortex. *Surviving small-volume correction at the cluster level.

the hypothesized impact of episodic prospection: both indices were greater for the Imagine than for the Estimate task (one-tailed paired-samples $t$ test: choice index, $t_{(11)}=2.2, p=0.025$; reward index, $t_{(11)}=2.25, p=0.023$; Fig. $2 B$ ). Thus, the results provide direct experimental evidence that episodic prospection can effectively attenuate temporal discounting.

This effect was more pronounced for episodes that were imagined with a higher emotional intensity, as indicated by positive correlations between the rating levels (i.e., 0-3) and the associated difference scores of delayed versus immediate options chosen at each level [Spearman's $\rho=0.41 \pm 0.16$ (mean \pm SEM); onesample $t$ test: $t_{(11)}=2.51, p=0.029$. Spearman rather than Pearson correlations were computed due to the ordinal nature of the rating scale]. Thus, participants were more likely to choose the delayed option with greater experienced emotional intensity. Indeed, episodes that were imagined with the highest emotional intensity were always followed by choices of the delayed option. In contrast, there was no such relationship between emotional ratings and choices for the Estimate task ( $\rho=$ $0.05 \pm 0.21 ; t_{(10)}=0.24, p=0.817$ ).

We further characterized the effect of episodic prospection by examining its relationship with individual differences in the predisposition to care about the future. This trait was assessed with the Consideration of Future Consequences scale (Strathman et al., 1994), while the effect size of episodic prospection was quantified by the ratio of the reward indices in the Imagine versus the Estimate task. These measures were negatively correlated (Pear-
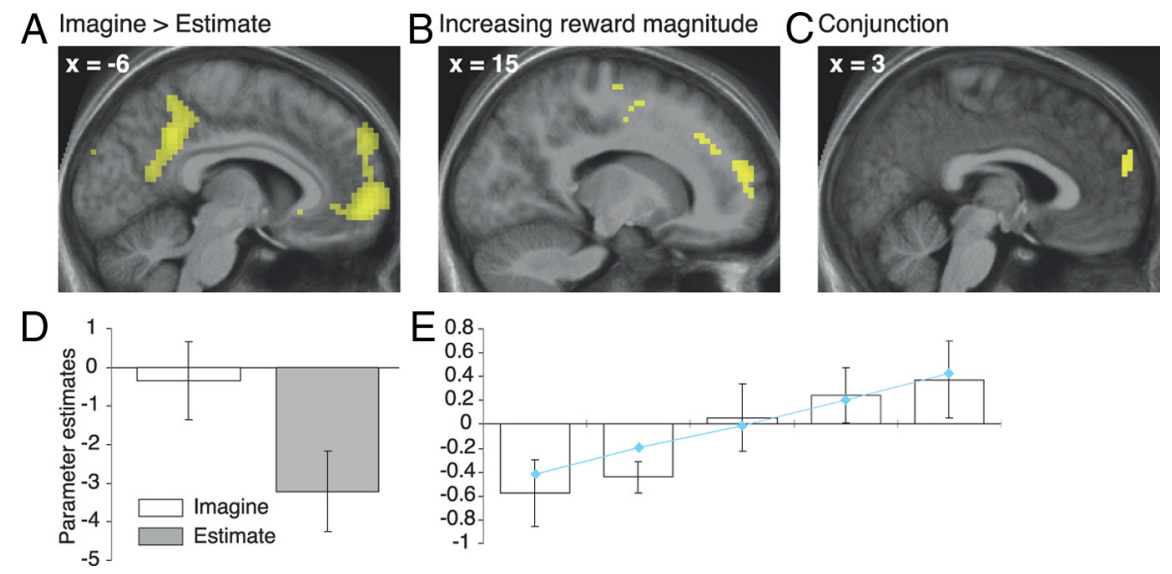

Figure 3. Neuroimaging results. $\boldsymbol{A}-\boldsymbol{C}, \mathrm{BOLD}$ signal increases for the Imagine versus the Estimate task $(\boldsymbol{A})$, the parametric modulation by reward magnitude in the Imagine versus Estimate task $(\boldsymbol{B})$, and the conjunction of both contrasts $(\boldsymbol{C})$. (For display purposes, the SPM is thresholded at $p<0.001$, uncorrected, at least 10 contiguous voxels.) $\boldsymbol{D}, \boldsymbol{E}$, Parameter estimates, averaged from a $5 \mathrm{~mm}$ sphere centered on the peak of the conjunction contrast, for the task effect $(\boldsymbol{D})$ and the parametric modulation of the Imagine task by reward magnitude $(\boldsymbol{E})$. Connected blue diamonds indicate the mean-centered natural logarithm of the reward magnitudes. Error bars reflect the SEM.

son's $r_{(12)}=-0.7, p=0.01$; Fig. $\left.2 C\right)$. Thus, directed episodic prospection was especially effective for those who are usually more indifferent toward the long-term consequences of their actions.

\section{Engagement of mrPFC during episodic prospection and reward representation}

The Imagine versus the Estimate task was associated with greater BOLD signal in a network of brain areas, including bilateral parahippocampal gyri, retrosplenial cortices, and precuneus (Table 1 ), that has previously been implicated in the construction of coherent scenes (e.g., during spatial navigation, autobiographical memory retrieval, or the imagination of fictitious events) (Has- 
Table 2. Regions in which BOLD signal showed significantly greater modulation by reward magnitude in the Imagine versus Estimate task

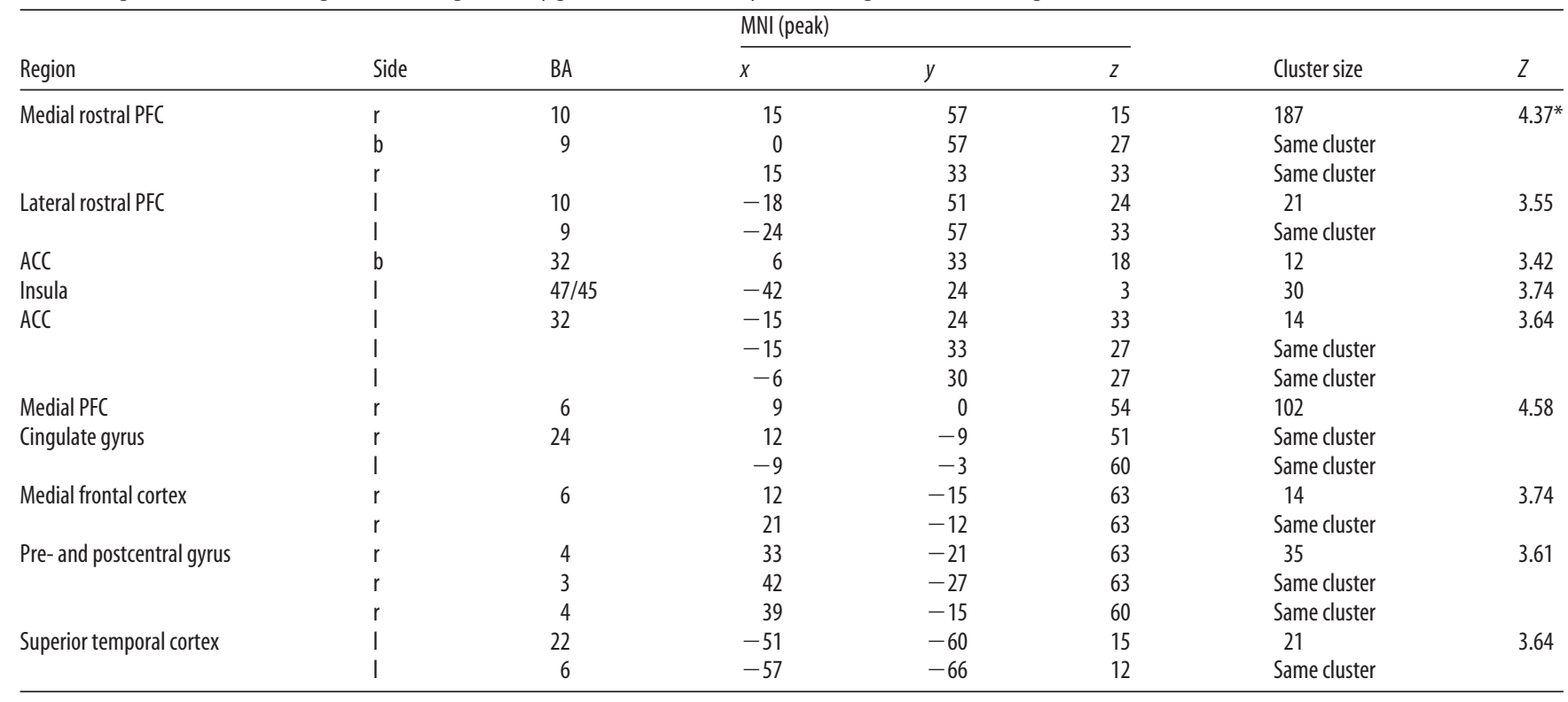

Thresholded at $p<0.001$, uncorrected, with a minimum cluster size of 10 contiguous voxels. Brodmann areas (BA) are approximate. b, Bilateral; I, left; r, right; ACC, anterior cingulate cortex. *Surviving small-volume correction at the cluster level.

Table 3. Results from the conjunction analysis: regions showing significant BOLD signal increase for both the task contrast (Imagine $>$ Estimate) and the contrast of the parametric modulations of the tasks by reward magnitude

\begin{tabular}{|c|c|c|c|c|c|c|c|}
\hline \multirow[b]{2}{*}{ Region } & \multirow[b]{2}{*}{ Side } & \multirow[b]{2}{*}{$\mathrm{BA}$} & \multicolumn{3}{|c|}{ MNI (peak) } & \multirow[b]{2}{*}{ Cluster size } & \multirow[b]{2}{*}{$Z$} \\
\hline & & & $x$ & $y$ & $Z$ & & \\
\hline \multirow[t]{2}{*}{ Medial rostral PFC } & $\mathrm{b}$ & $9 / 10$ & -3 & 57 & 30 & 26 & \multirow[t]{2}{*}{$3.60^{*}$} \\
\hline & $\mathrm{b}$ & 10 & 3 & 57 & 18 & Same cluster & \\
\hline \multirow[t]{2}{*}{ Dorsal temporal cortex } & I & 22 & -51 & -60 & 15 & 15 & \multirow[t]{2}{*}{3.64} \\
\hline & I & 19 & -57 & -66 & 12 & Same cluster & \\
\hline
\end{tabular}

Thresholded at $p<0.001$, uncorrected, with a minimum cluster size of 10 contiguous voxels. Brodmann areas (BA) are approximate. b, Bilateral; l, left. *Surviving small-volume correction at the cluster level.

sabis and Maguire, 2007; Spreng et al., 2009). Importantly, the contrast also revealed increased BOLD signal in mrPFC (surviving small-volume correction at the cluster level; Fig. $3 A$ ). We hypothesized that if activation in this region reflects the reward magnitude of imagined episodes, at least some part of it should also yield BOLD signal changes as a function of the amount of money that had to be considered (i.e., a parametric effect of reward magnitude). Therefore, we examined which brain areas showed a stronger link between BOLD signal and monetary value during the Imagine task than during the Estimate task. This contrast revealed signal changes in anterior cingulate, left anterior insula, right precentral gyrus, left superior temporal cortex, and prefrontal cortex (PFC) (Table 2). As expected, the most prominent prefrontal cluster was within mrPFC (surviving smallvolume correction at the cluster level; Fig. $3 B$ ). A conjunction analysis, using the minimum statistic approach (Nichols et al., 2005), showed that two clusters were jointly activated by the contrasts of the Imagine versus Estimate task, and the parametric effects of reward magnitude. One of these clusters was located in dorsal temporal cortex. Crucially, the other one was situated within mrPFC (surviving small-volume correction at the cluster level) (Fig. 3C-E; Table 3). (By contrast, activation in this region during the Imagine task did not reflect the duration of the delay, the feeling of experiencing an event, or the emotional intensity, even at a liberal threshold of $p<0.05$, uncorrected.) Therefore, our data demonstrate that some of the mrPFC activation observed during the imagination of future scenarios can indeed be associated with the reward magnitude of the envisaged episodes.
mrPFC BOLD signal and mrPFC-hippocampal coupling predict attenuated discounting

Lesions including mrPFC can lead to short-sighted behavior (Bechara and Damasio, 2008; Sellitto et al., 2010), and have been associated with a foreshortened personal future time perspective (Fellows and Farah, 2005). The current data suggest that this region might support perspicacious future-oriented behavior by processing the reward magnitude of prospective episodes. In accordance with this account, there was a tight link between BOLD signal sensitivity for imagined reward magnitudes and the attenuating effect of episodic prospection. Reward sensitivity was defined as the ratio of the parameter estimates for highest versus lowest rewards (from a $5 \mathrm{~mm}$ radius sphere at the conjunction peak), whereas the behavioral effect size was assessed by the ratio of the reward indices in the Imagine versus the Estimate task. Across participants, these measures were positively correlated

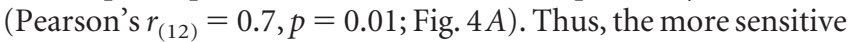
$\mathrm{mrPFC}$ was to the magnitude of imagined rewards, the greater was the impact of envisaging future rewards on temporal discounting. [This association was also significant when using Spearman's $\rho(r=0.671 ; p=0.017)$, indicating that it did not merely reflect the contribution of potential outliers.] In contrast, there was no such correlation for the dorsal temporal cluster (Pearson's $r_{(12)}=-0.08, p=0.81$; Fig. $4 B$ ), suggesting that this effect was specific to mrPFC.

We hypothesized that if $\mathrm{mrPFC}$ represents the reward magnitude of imagined events, it might interact with the $\mathrm{HC}$, a region critically involved in mentally constructing those 

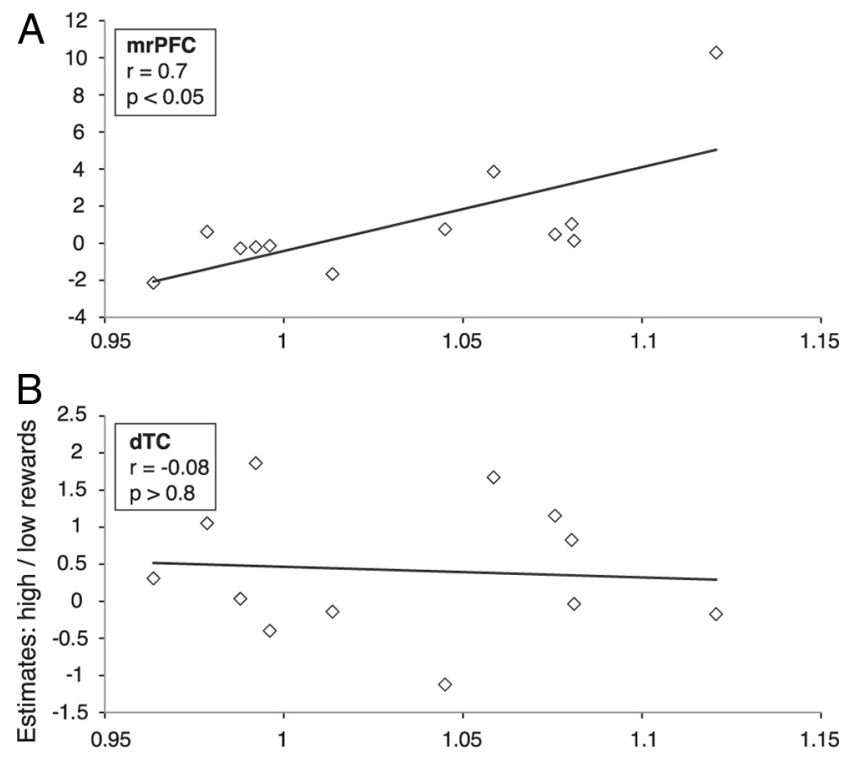

Ratio of reward indices: imagine / estimate

$\mathrm{C}_{\text {PPI seed }}$ Correlation with episodic-prospection effect
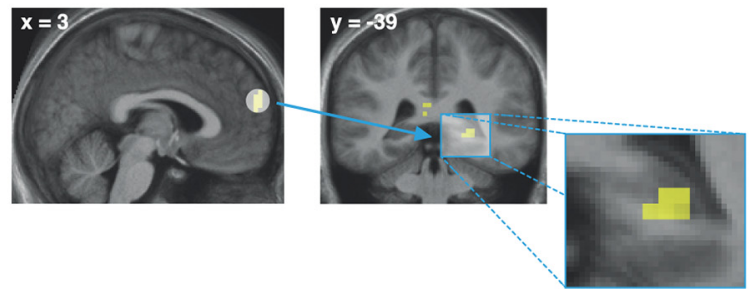

Figure 4. Brain-behavior relationships. $A, B$, Correlations between (1) BOLD signal sensitivity to reward magnitude and (2) the ratios of reward indices in the Imagine versus the Estimate task. C, A PPI analysis revealed stronger coupling between mrPFC and right hippocampus during the Imagine task for those participants who also exhibited a greater effect of episodic prospection on discounting. (For display purposes, the PPI image is thresholded at $p<0.005$, uncorrected, at least 5 contiguous voxels.) dTC, Dorsal temporal cortex.

events (Hassabis and Maguire, 2007). Lesions to this region diminish the ability to envisage coherent episodes (Hassabis et al., 2007), and a greater coupling with prefrontal regions has recently been linked to less impulsive monetary choices (Peters and Büchel, 2010). Therefore, a PPI analysis (Friston et al., 1997) was performed to reveal those brain regions showing greater functional coupling with $\mathrm{mrPFC}$ during the Imagine than during the Estimate task. We then conducted a regression analysis between the coupling parameters within $\mathrm{HC}$ and the effect of episodic prospection on discounting. This analysis revealed stronger connectivity between mrPFC and HC $(x=$ 27, $y=-39, z=0 ; Z=3.1$; surviving small-volume correction at the cluster level) for those participants who also showed a greater effect size (Fig. 4C).

\section{mrPFC BOLD signal during episodic prospection predicts subsequent farsighted choices}

Sensitivity of mrPFC BOLD signal to reward magnitudes as well as increased mrPFC-HC coupling explained some of the individual differences in the attenuating effect of episodic prospection. However, if $\mathrm{mrPFC}$ is involved in mediating this effect, BOLD signal changes in this region might also predict individual decisions on a trial-by-trial basis. We therefore per- formed a subsequent choice analysis that contrasted trials of the Imagine task that were followed by decisions for the delayed versus the immediate option. Parametric regressors controlled for effects of reward magnitudes associated with each of those two trial types. The analysis revealed just one cluster, which was located in dorsal mrPFC $(9,48,39$; Brodmann area 9; $Z=3.43$; surviving small-volume correction at the cluster level; Fig. 5), adjacent to the cluster associated with the processing of reward magnitude during the Imagine task. For the Estimate task, by contrast, mrPFC BOLD signal did not predict subsequent choices (even at a lenient threshold of $p<$ 0.05 , uncorrected, within the whole mrPFC mask).

\section{Discussion}

This study provides new insights into the adaptive function of episodic prospection and its neuroanatomical basis. Imagining future episodes has been hypothesized to bias decisions toward farsighted choices by conveying the reward value of the envisaged event (Boyer, 2008). Consistently, participants were more likely to choose a delayed but larger reward instead of an immediate but smaller reward, if they had previously imagined the consumption of the delayed reward. Thus, episodic prospection effectively attenuated temporal discounting. Our data indicate that participants were especially likely to delay the receipt of a reward after having imagined its consumption with greatest emotional intensity. This anticipated intensity might thus constitute an intrinsic source of utility that influences the valuation of the delayed choice (Berns et al., 2007). Of note, also the control condition prompted the participants to consider the reward magnitude of the delayed options with respect to the scenarios. Thus, this effect is unlikely to just reflect increased attention toward the delayed option or greater salience of its higher purchasing power.

The impact of episodic prospection was particularly strong for those individuals who are usually less prone to consider the future consequences of their actions. This variable has been shown to mediate, among others, socioeconomic inequalities in health behaviors (Adams, 2009), the relationship between beliefs regarding environmental consequences of commuting by car and the preference to take public transport (Joireman et al., 2004), and between planning and actually quitting smoking (Kovač and Rise, 2007). Directed episodic prospection might accordingly have a beneficial impact on decision making in these domains.

Importantly, we provide evidence that mrPFC might directly support the attenuating effect of episodic prospection. Activation in this region was associated with the imagination of future episodes as well as with the considered reward magnitude. Note that we observed this functional overlap despite the great spatial heterogeneity of regions previously implicated in the processing of subjective values [which included considerably more inferior, orbitofrontal areas (Plassmann et al., 2007)]. Thus, some of the BOLD signal changes consistently linked to the simulation of future episodes (Addis et al., 2007; Szpunar et al., 2007; D’Argembeau et al., 2008; Spreng et al., 2009) might indeed reflect the reward value of the envisaged events. However, given the substantial fine-grained functional specialization within mrPFC (Gilbert et al., 2006, 2010; Krueger et al., 2007), it is noteworthy that BOLD signal changes in other parts were more selectively associated with either episodic prospection or reward representation. These subregions might be engaged for processing different aspects of episodic prospection, such as the self-referential nature of 


\section{Subsequent choice: delayed $>$ immediate}
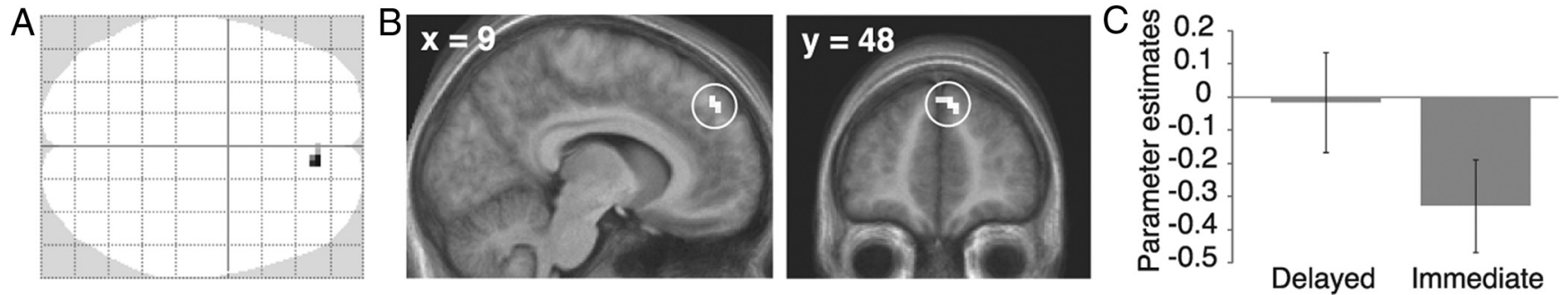

Figure 5. Subsequent choice analysis. $A, B$, The only region showing greater BOLD signal during trials of the Imagine task that were followed by choices of the delayed versus immediate option. (For display purposes, the SPM is thresholded at $p<0.001$, uncorrected, at least 10 contiguous voxels.) $C$, Parameter estimates, averaged from a 5 mm sphere centered on the peak of the subsequent choice effect. Error bars indicate the SEM.

the imagined scenes (Abraham et al., 2008; Benoit et al., 2010; D’Argembeau et al., 2010).

The reward representation supported by mrPFC seems to mediate the impact of episodic prospection on farsighted decisions: there was a tight link between BOLD signal sensitivity for reward magnitude and the extent of attenuated discounting. Those individuals who exhibited stronger BOLD signal increases while imagining highest versus lowest rewards also showed reduced discounting of those rewards. Thus, the representation of reward magnitude might support the experience of the (undiscounted) affective impact of future episodes. This, in turn, might add weight to the valuation of the choice leading to the envisaged event. Moreover, BOLD signal in dorsal mrPFC predicted subsequent choices on a trial-by-trial basis, indicating a fundamental role of this region in supporting farsighted decisions. The dorsal medial prefrontal surface has previously been argued to represent the value of potential future actions (Amodio and Frith, 2006). For example, activation in this region is correlated with the expected probability of monetary gain (Knutson et al., 2005), and it increases when participants monitor the outcome of self-selected actions (Walton et al., 2004). Dorsal mrPFC might accordingly use the representation of imagined rewards to guide adaptive behavior.

Previous research has shown that the representation of subjective value in medial frontal cortex (including parts of mrPFC) can be modulated to achieve farsighted outcomes. For example, such a "value signal" was shown to primarily reflect the taste of food options for control participants, whereas it also incorporated the health ratings for participants whose decisions were influenced by dietary considerations (Hare et al., 2009). This shift of value representation seemed to be instantiated via control exerted by dorsolateral PFC. The current study elucidates a further modulatory mechanism of reward representation in mrPFC. Those participants who showed the greatest impact of episodic prospection on monetary choices also exhibited the strongest coupling between mrPFC and hippocampus.

Though these two regions are consistently coactivated during episodic prospection (Spreng et al., 2009), they seem to support distinct processes. Hippocampal lesions lead to specific impairments in imagining fictitious events: the envisaged episodes lack spatial coherence (Hassabis et al., 2007). This region has therefore been argued to be critically involved in scene construction, i.e., the generation and maintenance of coherent and complex events (Hassabis and Maguire, 2007; see also Bird and Burgess, 2008). Accordingly, the effect of episodic prospection might be based on an interaction between regions supporting the construction of events (i.e., HC) and the representation of the events' reward magnitude (i.e.,
mrPFC). This neural circuitry may support a similar function in the acquisition of conceptual knowledge, where mrPFC has been linked to the evaluation of associative information bound by the HC (Kumaran et al., 2009). The interaction of these areas might be grounded in their direct anatomical connections (Carmichael and Price, 1995; Thierry et al., 2000).

Consistent with the present findings, a recent study reported a relationship between attenuated discounting and hippocampalmedial prefrontal coupling while participants spontaneously engaged in episodic prospection (Peters and Büchel, 2010). However, in that study, HC was coupled with the anterior cingulate cortex rather than mrPFC. This may reflect crucial differences in the experimental designs: in contrast to the present study, there was no direct correspondence between the amount of money that had to be considered and the putatively imagined episode. Moreover, the control condition did not share the semantic retrieval demands of episodic prospection. Instead, the link between episodic prospection and attenuated discounting was primarily based on questionnaires assessing the degree of spontaneous imagery during the episodic condition. Finally, the data were obtained during a period in which participants had to choose between the delayed and immediate reward options, whereas the current neuroimaging analysis concentrated on a preceding time period that required the direct mental experience of the rewards' full impact.

This latter difference might also account for the distinct functional properties of mrPFC observed in the two studies. Peters and Büchel (2010) reported a greater correlation during the episodic than during the control condition between BOLD signal in mrPFC (among other regions) and subjective (i.e., discounted) value. In their study, processes supporting episodic imagery likely coincided with valuation processes required for the imminent decision. By contrast, our study yielded a stronger correlation during episodic prospection than in the control condition between mrPFC activation and the absolute reward magnitude of the imagined events. Here, participants focused on the experience of the future episode without having to prepare their choice at the same time. Thus, in situations of pure episodic prospection, mrPFC might support the experience of the imagined event's undiscounted reward value. This experience may mediate the striking impact of episodic prospection on farsighted decisions.

What is the mechanism by which mrPFC might represent reward values of imagined episodes? Rostral PFC features a higher spine density and number of dendritic spines per cell than do comparable cortical areas (Jacobs et al., 2001). These anatomical properties make this region especially likely to support the integration of multiple inputs (Ramnani and Owen, 2004). Spe- 
cifically, mrPFC is thought to incorporate autonomic information from anterior insula, anterior cingulate cortex (which were coactivated in the present study), and other regions to generate a representation of the current feeling state (Critchley, 2005). This state has been suggested to constitute a "somatic marker" that biases decisions by linking potential affective consequences with choice options (Bechara and Damasio, 2008). mrPFC might accordingly be involved in the attenuating effect of episodic prospection on temporal discounting: it might process the utility of choices leading to imagined episodes, thereby motivating farsighted decisions. Consistent with this account, lesions including mrPFC can lead to exaggerated temporal discounting (Sellitto et al., 2010).

To conclude, the human faculty of envisaging possible future scenarios seems to serve an adaptive function: it effectively motivates decisions in the present which will only be advantageous in the future. Our data indicate that mrPFC plays a pivotal role in mediating this effect by representing the reward that a prospective episode would hold. We further demonstrate that this effect is particularly strong for those individuals who are typically more indifferent toward the future consequences of their actions. A better understanding of this mechanism might thus help to optimize everyday decisions that have economic, environmental, and public health-related consequences.

\section{References}

Abraham A, Schubotz RI, von Cramon DY (2008) Thinking about the future versus the past in personal and non-personal contexts. Brain Res 1233:106-119.

Adams J (2009) The mediating role of time perspective in socio-economic inequalities in smoking and physical activity in older English adults. J Health Psychol 14:794-799.

Addis DR, Wong AT, Schacter DL (2007) Remembering the past and imagining the future: common and distinct neural substrates during event construction and elaboration. Neuropsychologia 45:1363-1377.

Amodio DM, Frith CD (2006) Meeting of minds: the medial frontal cortex and social cognition. Nat Rev Neurosci 7:268-277.

Atance CM, O’Neill DK (2001) Episodic future thinking. Trends Cogn Sci 5:533-539.

Bechara A, Damasio AR (2008) The somatic marker hypothesis: a neural theory of economic decision. Games Econ Behav 52:336-372.

Benoit RG, Gilbert SJ, Volle E, Burgess PW (2010) When I think about me and simulate you: medial rostral prefrontal cortex and self-referential processes. Neuroimage 50:1340-1349.

Bernoulli D (1738) [Exposition of a new theory on the measurement of risk]. Translated (1954) by L Sommer. Econometrica 22:23-36.

Berns GS, Laibson D, Loewenstein G (2007) Intertemporal choice-toward an integrative framework. Trends Cogn Sci 11:482-488.

Bickel WK, Pitcock JA, Yi R, Angtuaco EJ (2009) Congruence of BOLD response across intertemporal choice conditions: fictive and real money gains and losses. J Neurosci 29:8839-8846.

Bird CM, Burgess N (2008) The hippocampus and memory: insights from spatial processing. Nat Rev Neurosci 9:182-194.

Bird CM, Capponi C, King JA, Doeller CF, Burgess N (2010) Establishing the boundaries: the hippocampal contribution to imagining scenes. J Neurosci 30:11688-11695.

Boettiger CA, Mitchell JM, Tavares VC, Robertson M, Joslyn G, D’Esposito M, Fields HL (2007) Immediate reward bias in humans: fronto-parietal networks and a role for the catechol-O-methyltransferase $158(\mathrm{Val} / \mathrm{Val})$ genotype. J Neurosci 27:14383-14391.

Boyer P (2008) Evolutionary economics of mental time travel? Trends Cogn Sci 12:219-224.

Carmichael ST, Price JL (1995) Limbic connections of the orbital and medial prefrontal cortex in macaque monkeys. J Comp Neurol 363:615-641.

Critchley HD (2005) Neural mechanisms of autonomic, affective, and cognitive integration. J Comp Neurol 493:154-166.

D’Argembeau A, Xue G, Lu ZL, Van der Linden M, Bechara A (2008) Neural correlates of envisioning emotional events in the near and far future. Neuroimage 40:398-407.
D’Argembeau A, Stawarczyk D, Majerus S, Collette F, Van der Linden M, Feyers D, Maquet P, Salmon E (2010) The neural basis of personal goal processing when envisioning future events. J Cogn Neurosci 22:17011713.

Fellows LK, Farah MJ (2005) Dissociable elements of human foresight: a role for the ventromedial frontal lobes in framing the future, but not in discounting future rewards. Neuropsychologia 43:1214-1221.

Friston KJ, Holmes AP, Worsley KJ, Poline JP, Frith CD, Frachowiak RS (1995) Statistical parametric maps in functional imaging: a general linear approach. Hum Brain Mapp 2:189-210.

Friston KJ, Buechel C, Fink GR, Morris J, Rolls E, Dolan RJ (1997) Psychophysiological and modulatory interactions in neuroimaging. Neuroimage 6:218-229.

Gilbert SJ, Spengler S, Simons JS, Steele JD, Lawrie SM, Frith CD, Burgess PW (2006) Functional specialization within rostral prefrontal cortex (area 10): a meta-analysis. J Cogn Neurosci 18:932-948.

Gilbert SJ, Henson RN, Simons JS (2010) The scale of functional specialization within human prefrontal cortex. J Neurosci 30:1233-1237.

Green L, Myerson J (2004) A discounting framework for choice with delayed and probabilistic rewards. Psychol Bull 130:769-792.

Hare TA, Camerer CF, Rangel A (2009) Self-control in decision-making involves modulation of the vmPFC valuation system. Science 324:646-648.

Hassabis D, Maguire EA (2007) Deconstructing episodic memory with construction. Trends Cogn Sci 11:299-306.

Hassabis D, Kumaran D, Vann SD, Maguire EA (2007) Patients with hippocampal amnesia cannot imagine new experiences. Proc Natl Acad Sci U S A 104:1726-1731.

Jacobs B, Schall M, Prather M, Kapler E, Driscoll L, Baca S, Jacobs J, Ford K, Wainwright M, Treml M (2001) Regional dendritic and spine variation in human cerebral cortex: a quantitative Golgi study. Cereb Cortex 11:558-571.

Johnson MW, Bickel WK (2002) Within-subject comparison of real and hypothetical money rewards in delay discounting. J Exp Anal Behav 77:129-146.

Joireman JA, Van Lange PAM, Van Vugt M (2004) Who cares about the environmental impact of cars?: Those with an eye toward the future. Environ Behav 36:187-206.

Kable JW, Glimcher PW (2007) The neural correlates of subjective value during intertemporal choice. Nat Neurosci 10:1625-1633.

Knutson B, Taylor J, Kaufman M, Peterson R, Glover G (2005) Distributed neural representation of expected value. J Neurosci 25:4806-4812.

Kovač VB, Rise J (2007) The relation between past behavior, intention, planning, and quitting smoking: the moderating effect of future orientation. J Appl Biobehav Res 12:82-100.

Krueger F, Moll J, Zahn R, Heinecke A, Grafman J (2007) Event frequency modulates the processing of daily life activities in human medial prefrontal cortex. Cereb Cortex 17:2346-2353.

Kumaran D, Summerfield JJ, Hassabis D, Maguire EA (2009) Tracking the emergence of conceptual knowledge during human decision making. Neuron 63:889-901.

Madden GJ, Begotka AM, Raiff BR, Kastern LL (2003) Delay discounting of real and hypothetical rewards. Exp Clin Psychopharmacol 11:139-145.

Maldjian JA, Laurienti PJ, Kraft RA, Burdette JH (2003) An automated method for neuroanatomic and cytoarchitectonic atlas-based interrogation of fMRI data sets. Neuroimage 19:1233-1239.

Montague PR, King-Casas B, Cohen JD (2006) Imaging valuation models in human choice. Annu Rev Neurosci 29:417-448.

Nichols T, Brett M, Andersson J, Wager T, Poline JB (2005) Valid conjunction inference with the minimum statistic. Neuroimage 25:653-660.

Peters J, Büchel C (2009) Overlapping and distinct neural systems code for subjective value during intertemporal and risky decision making. J Neurosci 29:15727-15734.

Peters J, Büchel C (2010) Episodic future thinking reduces reward delay discounting through an enhancement of prefrontal-mediotemporal interactions. Neuron 66:138-148.

Pine A, Seymour B, Roiser JP, Bossaerts P, Friston KJ, Curran HV, Dolan RJ (2009) Encoding of marginal utility across time in the human brain. J Neurosci 29:9575-9581.

Plassmann H, O'Doherty J, Rangel A (2007) Orbitofrontal cortex encodes willingness to pay in everyday economic transactions. J Neurosci 27:9984-9988. 
Ramnani N, Owen AM (2004) Anterior prefrontal cortex: insights into function from anatomy and neuroimaging. Nat Rev Neurosci 5:184-194.

Rushworth MF, Behrens TE (2008) Choice, uncertainty and value in prefrontal and cingulate cortex. Nat Neurosci 11:389-397.

Schacter DL, Addis DR, Buckner RL (2007) Remembering the past to imagine the future: the prospective brain. Nat Rev Neurosci 8:657-661.

Sellitto M, Ciaramelli E, di Pellegrino G (2010) Myopic discounting of future rewards after medial orbitofrontal damage in humans. J Neurosci 30:16429-16436.

Sharot T, Riccardi AM, Raio CM, Phelps EA (2007) Neural mechanisms mediating optimism bias. Nature 450:102-105.

Spreng RN, Mar RA, Kim AS (2009) The common neural basis of autobiographical memory, prospection, navigation, theory of mind, and the default mode: a quantitative meta-analysis. J Cogn Neurosci 21:489-510.
Strathman A, Gleicher F, Boninger DS, Edwards CS (1994) The consideration of future consequences: weighing immediate and distant outcomes of behavior. J Pers Soc Psychol 66:742-752.

Suddendorf T, Corballis MC (2007) The evolution of foresight: What is mental time travel, and is it unique to humans? Behav Brain Sci 30:299-313.

Szpunar KK, Watson JM, McDermott KB (2007) Neural substrates of envisioning the future. Proc Natl Acad Sci U S A 104:642-647.

Thierry AM, Gioanni Y, Dégénétais E, Glowinski J (2000) Hippocampoprefrontal cortex pathway: anatomical and electrophysiological characteristics. Hippocampus 10:411-419.

Walton ME, Devlin JT, Rushworth MF (2004) Interactions between decision making and performance monitoring within prefrontal cortex. Nat Neurosci 7:1259-1265. 